\title{
BMJ Open Towards a safer culture: implementing multidisciplinary simulation-based team training in New Zealand operating theatres - a framework analysis
}

\author{
Tanisha Jowsey (D) , ${ }^{1}$ Peter Beaver, ${ }^{1}$ Jennifer Long, ${ }^{1}$ Ian Civil, ${ }^{2,3}$ A L Garden, ${ }^{4}$ \\ Kaylene Henderson, ${ }^{1,5}$ Alan Merry, ${ }^{6}$ Carmen Skilton, ${ }^{1}$ Jane Torrie, ${ }^{5,6}$ \\ Jennifer Weller (D) ${ }^{1,5}$
}

To cite: Jowsey T, Beaver P, Long J, et al. Towards a safer culture: implementing multidisciplinary simulationbased team training in New Zealand operating theatres - a framework analysis. BMJ Open 2019;9:e027122. doi:10.1136/ bmjopen-2018-027122

- Prepublication history and additional material for this paper are available online. To view these files, please visit the journal online (http://dx.doi. org/10.1136/bmjopen-2018027122).

Received 25 January 2019 Revised 11 July 2019 Accepted 25 September 2019

Check for updates

(C) Author(s) (or their employer(s)) 2019. Re-use permitted under CC BY-NC. No commercial re-use. See rights and permissions. Published by BMJ.

For numbered affiliations see end of article.

Correspondence to Professor Jennifer Weller; j.weller@auckland.ac.nz

\section{ABSTRACT}

Aim NetworkZ is a simulation-based multidisciplinary teamtraining programme designed to enhance patient safety by improving communication and teamwork in operating theatres (OTs). In partnership with the Accident Compensation Corporation, its implementation across New Zealand (NZ) began in 2017. Our aim was to explore the experiences of staff - including the challenges they faced - in implementing NetworkZ in NZ hospitals, so that we could improve the processes necessary for subsequent implementation. Method We interviewed staff from five hospitals involved in the initial implementation of NetworkZ, using the Organising for Quality model as the framework for analysis. This model describes embedding successful quality improvement as a process of overcoming six universal challenges: structure, infrastructure, politics, culture, motivation and learning.

Results Thirty-one people participated. Structural support within the hospital was considered essential to maintain staff enthusiasm, momentum and to embed the programme. The multidisciplinary, simulation-based approach to team training was deemed a fundamental infrastructure for learning, with participants especially valuing the realistic in situ simulations and educational support. Participants reported positive changes to the OT culture as a result of NetworkZ and this realisation motivated its implementation. In sites with good structural support, NetworkZ implementation proceeded quickly and participants reported rapid cultural change towards improved teamwork and communication in their OTs. Conclusion Implementation challenges exist and strategies to overcome these are informing future implementation of NetworkZ. Embedding the programme as business as usual across a nation requires significant and sustained support at all levels. However, the potential gains in patient safety and workplace culture from widespread multidisciplinary team training are substantial. Trial registration number

ACTRN12617000017325.

\section{INTRODUCTION}

Background

Outcomes for patients undergoing surgery are not always as intended. There is good
Strengths and limitations of this study

- A strength of this study is the focus on implementation of a simulation-based team training programme across an entire country, lessons learnt and potential lessons for others.

- A further strength is the application of the Organising for Quality framework to explore implementation challenges.

- One limitation is that interview participants were volunteers who had agreed both to the interview, and to taking part in the implementation of the NetworkZ programme in their hospital, and were likely to be invested in its success.

- A further limitation is that this study has only reported on the perspectives of local hospital staff charged with the implementation of the programme, and has not included the perspectives of those charged with the overall implementation at the national level.

- The extent to which implementation lessons from a national team-training programme in a country of five million can be scaled up to larger populations remains to be tested.

evidence that failures in teamwork and communication commonly play a role in adverse events. ${ }^{12}$ Lingard et $a \vec{l}$ identified that $30 \%$ of team interactions in the operating theatre (OT) failed in some way to convey the correct message. For several decades now, there have been calls for multidisciplinary team training in healthcare. ${ }^{4}{ }^{5}$ Over these decades, we have collected evidence showing that such training improves team function, organisational processes and patient outcomes. ${ }^{6-8}$ Yet for the most part, team training for surgical teams is far from established as usual practice.

In 2016, we set out to implement multidisciplinary simulation-based team training as business as usual for OT teams in all New Zealand (NZ) public hospitals over a 5-year 


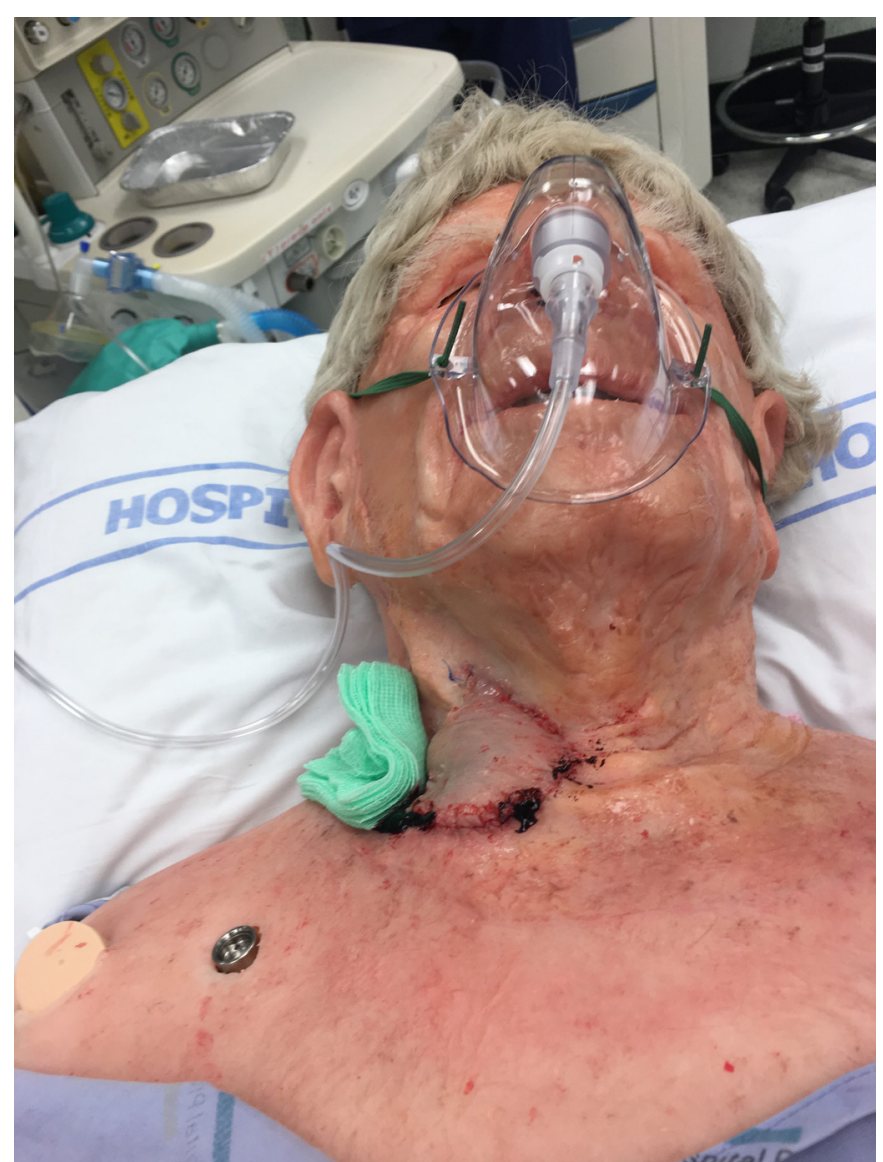

Figure 1 Simulated patient for NetworkZ training.

period. We called our programme 'NetworkZ.' There are 20 District Health Boards (DHBs) in NZ and an estimated 5000 qualified OT staff, including anaesthetists, surgeons, nurses and anaesthetic assistants. We divided these 20 DHBs into four cohorts, matched for DHB size, and began introducing the training to one cohort per year. Our implementation goal is that DHBs will independently run their own NetworkZ courses after 12 months of central support, and that all NZ OT staff will attend a NetworkZ course at least once in 5 years. This

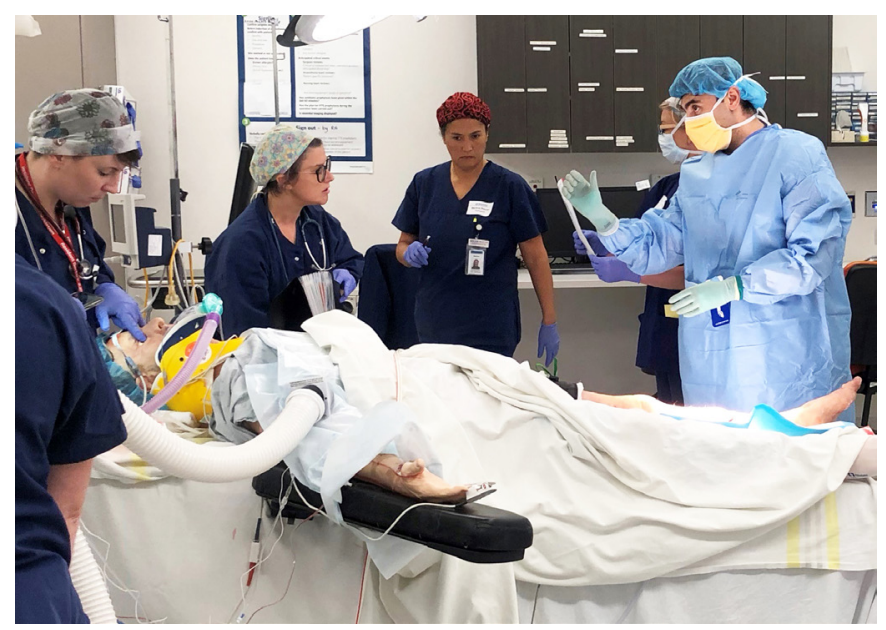

Figure 2 Multidisciplinary in situ simulation training underway. paper assesses the local experiences of implementation, with a focus on the challenges that were experienced and how these were overcome.

\section{NetworkZ programme}

NetworkZ was developed by a multidisciplinary team at the University of Auckland for all OT staff. A NetworkZ feasibility study demonstrated measurable improvements in communication in 20 multidisciplinary OT teams. ${ }^{9}$ Each NetworkZ training session is held in situ within a regular OT with the usual staff. The scenarios use a Laerdal 3G manikin fitted with a range of scenario-specific, customdesigned surgical models. Teams are briefed about the simulated patient and scenario, then they prepare for, and undertake, surgery (figure 1) (figure 2). A facilitated debrief follows. A half-day of training includes two simulations and debriefs. The training session also includes instruction in specific communication strategies focussing on closed loop communication, speaking up and structured recaps. A full description of the programme can be found at www.networkz.ac.nz

\section{NetworkZ in the context of the NZ health system}

In NZ, 20 DHBs are responsible for publicly-funded health services. ${ }^{10}$ In 2017, 330353 people (7.02\% of the population) received publicly-funded operations. ${ }^{11} \mathrm{NZ}$ publicly funds treatment for all victims of accidents, including injuries sustained during treatment in hospital. The fund is administered through the Accident Compensation Corporation (ACC). NZ has a Health Quality and Safety Commission (HQSC), which administers the Safe Surgery $\mathrm{NZ}$ programme ${ }^{12}$ - a national initiative to improve safety for surgical patients. ${ }^{13}$ NetworkZ sits within this context, funded by the Treatment Injury Prevention arm of ACC and in collaboration with the HQSC.

\section{Challenges in quality improvement implementation}

Internationally, efforts to improve patient safety have delivered 'mostly inconsistent and patchy results.' 1314 Jones $e t a l^{7}$ found 'team-training can result in transformational change in safety culture when the work environment supports transfer of learning to behaviour, ${ }^{, 7}$ but cautioned that successful implementation is dependent on an effective strategy for implementation.

Following an improvement science approach we adopted the Organising for Quality (OQ) theoretical model, ${ }^{15}$ for our approach to the national implementation of NetworkZ. The OQ model was developed from the study of many quality improvement initiatives across a range of healthcare organisations, looking for factors which facilitated or hindered implementation and sustained change. The authors of OQ describe embedding successful quality improvement as a process of overcoming six universal challenges: structure, infrastructure, politics, culture, motivation and learning. ${ }^{15}$ (table 1) Each challenge is interconnected and can work together synergistically in either a positive or negative direction. 
Table 1 Organising for Quality model of challenges in implementing quality improvement initiatives

\begin{tabular}{ll}
\hline Challenge & Comments \\
\hline $\begin{array}{l}\text { Structure } \\
\text { Infrastructure }\end{array}$ & $\begin{array}{l}\text { Organising, planning, coordination } \\
\text { Systems }\end{array}$ \\
Culture & $\begin{array}{l}\text { Developing a collective meaning, } \\
\text { importance and significance around quality } \\
\text { improvements }\end{array}$ \\
Politics & $\begin{array}{l}\text { Addressing and dealing with the politics of } \\
\text { change }\end{array}$ \\
Learning & $\begin{array}{l}\text { Encouraging learning that supports ongoing } \\
\text { improvement }\end{array}$ \\
Motivation & $\begin{array}{l}\text { Engaging and motivating people, linking } \\
\text { improvement to key values }\end{array}$ \\
\hline
\end{tabular}

\section{NetworkZ implementation strategy}

During the roll-out of NetworkZ, we were cognisant of the challenges described in the $\mathrm{OQ}$ model, and undertook specific activities to overcome each of these (see table 2).

At the time of writing this article, the NetworkZ programme was established in Cohort 1 and 2 DHBs, beginning in Cohort 3 and the process of engagement with senior executive groups in Cohort 4 had begun. All DHBs had signed up to the programme. Over 1000 OT staff had participated in a NetworkZ course, including over 200 who had attended an instructors training course.

\section{This study}

This study took place in 2017, during the early phase of NetworkZ implementation in Cohort 1.

The overall purpose of this study was to understand if the national implementation of NetworkZ was proceeding as expected, from the perspective of the local DHB staff tasked with its local implementation. To align with our implementation strategy, and explore the known major challenges in quality improvement, we used the $\mathrm{OQ}$ framework. Our aim was to identify at an early stage of implementation, the elements of our strategy that were working and those that required modification or refinement for subsequent Cohorts.

\section{METHODS}

Participants provided informed written consent prior to participation. The study is one component of a comprehensive evaluation plan, which includes patient outcome measures from a national database of surgical patients, measures of organisational change, interviews, team process measures (surveys and observations) and measures of safety culture.

\section{Interview guide}

We developed the interview guide for this study to explore experiences of programme setup, delivery, recruitment and scheduling by instructors, managers and project team members.

Draft interview questions were developed using an open framework to elicit novel ideas, but taking into account our experiences of early programme implementation. The questions were then reviewed by a team of three researchers (CS, JW, TJ), piloted and refined to more directly address issues of establishing the programme in the theatre environment, recruiting participants and delivering the training (online supplementary Appendix $1)$.

\section{Sample and recruitment}

All those involved in the implementation of NetworkZ in Cohort 1 were eligible to participate. We used a purposive sampling strategy aiming for a mix of participants with different roles in the implementation process. Some may have had more than one role across senior management, project teams and instructors. Potential participants were emailed an invitation to do a 20 to 30 min confidential telephone or video-conference interview. A follow-up email was sent to non-responders.

\section{Patient and public involvement}

There was no patient and public involvement in this study.

\section{Interviews and analysis}

The interviews were conducted by PB (medical sociologist) and CS (psychology graduate) between June 2017 and March 2018. All interviews were audio-recorded and transcribed verbatim by an independent typist who signed a non-disclosure statement prior to transcription.

The analysis took part in three stages. In the first stage, in between interviews, researchers $\mathrm{PB}$ and CS recorded notes (analytic memos), and discussed their findings and impressions with the immediate research team who are very experienced in qualitative research: JW (anaesthetist), KH (anaesthetic technician) and TJ (anthropologist). Transcripts were de-identified and entered into QSR NVivo 11 software for analysis.

In the second stage, following Gale $e t$ al, we undertook framework analysis. ${ }^{16}$ This is a form of qualitative analysis whereby researchers reorganise qualitative data (in this case, interview transcripts) into a new structure using a framework of themes. Using the original definitions of challenges we coded all data deductively, according to these challenges. ${ }^{15}$ During coding, the researchers met regularly to discuss the data and whether or not emerging findings fell within the OQ framework. Two researchers ( $\mathrm{PB}$ and $\mathrm{TJ}$ ) independently coded the first six transcripts and then compared and discussed coding to increase consistency and reliability in coding. PB then coded the remaining data and $\mathrm{TJ}$ checked $\mathrm{PB}$ 's coding.

The third stage involved synthesising and summarising data following the framework, which Gale et al refer to as 'charting. ${ }^{16}$ A report on the charting notes was presented to the research team for group discussion to check validity of conclusions. 


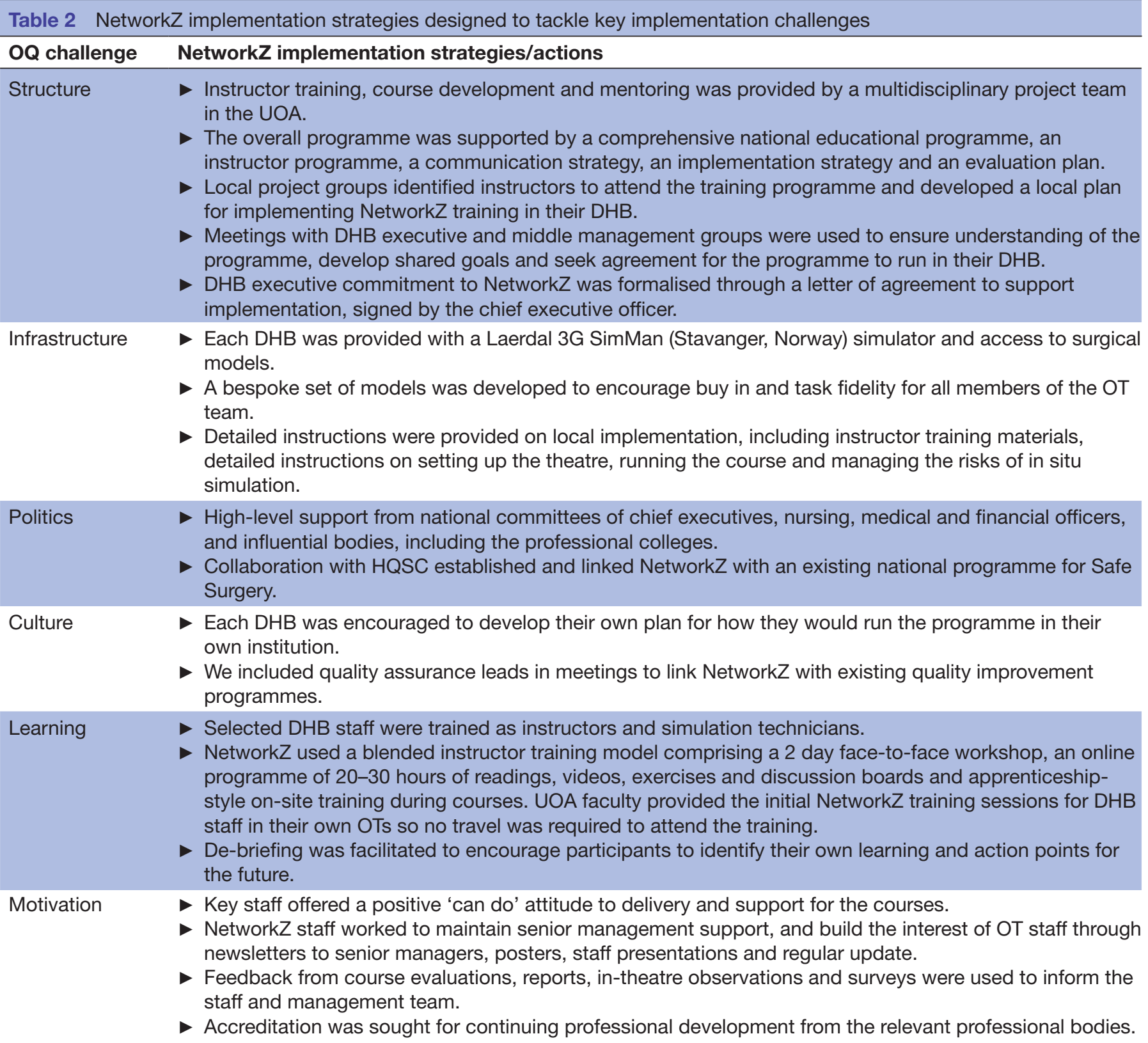

DHB, District Health Boards; HQSC, Health Quality and Safety Commission; OQ, Organising for Quality; OT, operating theatre; UOA, University of Auckland.

\section{RESULTS}

Of the 59 staff invited to participate, 31 were interviewed (23 females, 8 males). Of those 28 invited but not interviewed, 11 declined, and 17 either failed to respond or failed to agree to an interview date. Recruitment stopped after we conducted at least six interviews in each DHB, including two to four interviews with managers and project team members, and four to six interviews with instructors, and we reached data saturation against the OQ framework. Mean interview length was $34 \mathrm{~min}$. Of the 31 participants interviewed, 8 were on both the DHB Senior Executive group and the local NetworkZ project team, 6 were on both these groups and also instructing on NetworkZ and the remaining 17 were instructing on
NetworkZ. The professional groups were Management (7), Nursing (7), Anaesthesia (9), Surgery (4) and Anaesthesia Assistant (4).

The results are presented under the headings of the six universal challenges of the $\mathrm{OQ}$ framework.

\section{Structure}

It was evident that the setup structures in each DHB drove locally-distinct implementation processes that affected the morale of implementation staff, recruitment outcomes, the proportion of staff that could be trained, the decision to run half-day or full-day courses and the extent of the cultural transformation. 
DHB staff were mostly positive about their capacity to implement NetworkZ when DHB senior and middle management supported the programme.

'As a whole, we're really well supported. We know how important it is. And everybody here is invested and supported to do it so that's what's really good about it.' (DHB5, anaesthetist)

'Yeah, I think it's that multi-level engagement right from the start...about having the simulation champions and your DHB management, both executive and day-to-day level, all sat down, in the same room and have an explicit set of 'this is what the University is asking you to achieve'.' (DHB1, anaesthetist)

Good management support meant they could set training days in advance, staff were expected to participate, patients were not scheduled for the theatre designated for the training day and everyone looked for solutions to unexpected difficulties. Reported high levels of support facilitated rapid OT cultural change.

Implementation was challenging when the local supporting structures were difficult to create, where there was less will to risk any direct impact on surgical targets, instructors were not allocated sufficient time or a suite of existing programmes appeared to compete for resources.

'we're struggling to get funding for our time that we are giving. And people are doing things like taking annual leave days to attend things and that kind of thing.' (DHB1, nurse)

In one case, professional development half-days were used for training, with the benefit that staff were free of clinical duties and theatres were free. This was effective, although not all staff agreed with this use of existing education time, especially its impact on instructors who could not attend other concurrent education sessions.

\section{Infrastructure}

Participants described the manikins and scenarios as key to engagement and learning from the course. Participants said scenarios that were engaging for all team members, regardless of discipline, were critical to maximising their enthusiasm for training. The quality of the manikin and surgical models exceeded the expectations of many participants and this attention to detail was reported as positively contributing to team involvement. This detail included the life-like face masks, wound moulage and the significant challenges, for example, to control bleeding, presented by the surgical models.

Participants commented that staff appeared fully immersed in the simulations and behaving as they normally would during theatre. Many noted that the simulations were very good at engaging staff, including surgeons, some of whom had anticipated that they would have little active involvement.
'The simulation was more realistic than they thought it was going to be. They kind of forgot that it was a manikin.' (DHB4, nurse)

'The models are successful in engaging the surgeon in doing what he's asked which is actually what surgeons need to do, to be task focused and challenged at the same time.' (DHB2, surgeon)

\section{Culture}

Culture featured as an existing phenomena affecting implementation, but also as a phenomena altered by the programme. Existing global health service and locally-specific cultures influenced how NetworkZ would be interpreted by staff and incorporated into the OT schedule. Participants talked of longstanding hierarchical barriers and occupational silos within the OT, which were frustrating and difficult to change, but provided a rationale for engaging in team training.

'There is a culture in our theatres still where junior staff will say 'the swab count's not right' and clinicians will completely ignore them.' (DHB2, manager)

'We had an incident a couple of weeks ago...the patient kept having hypotension...nobody felt able to say 'there's a lot of bleeding'... the registrar was inhibited, and I suspect that's the case for the nursing staff also.' (DHB5, anaesthetist)

Participants observed that NetworkZ had already positively changed workplace culture, in the form of improved team relationships, communication, attitudes and workflow.

'I think there has been a bit more uptake of the briefing. Our theatre team certainly seems a bit happier and I don't know if that is a result of doing NetworkZ or something else. It's been a really stressful environment and I've really noticed over the past 6 months (since introduction of NetworkZ training), everything's a bit smoother and everyone is working together a bit better.' (DHB3, surgeon)

'It's created a lot of talk about things that they can do better and I think it has improved some relationships around the place and not just, I think nurse to nurse but nurse to doctor and things like that.' (DHB4, nurse)

'Anecdotally I'm hearing the conversation that people are happier to speak up, they're happier to raise a concern, they're challenging each other on a behaviour front if things are starting to get a bit heated in theatre.' (DHB5, manager)

The programme built on concurrent quality improvement initiatives designed to improve local safety culture, especially the WHO Surgical Safety Checklist briefing. These shifts were more specific to individuals in the larger DHBs where a small proportion of staff had been trained, and more widespread in the smaller sites with a greater saturation of training. 


\section{Politics}

To run a NetworkZ course, a full surgical team is required, so the training depends on the whole team participating. For some participants, establishing multidisciplinary training gave rise to political tensions due to the differences between the different professional groups' availability or willingness to participate, and perceptions of hierarchy. In almost all accounts the political tensions described by participants circled around recruiting surgeons. Non-surgeon participants voiced frustration that the training was often organised around the priorities of one group, which was perceived as counter to the NetworkZ goals of improved team collaboration.

Challenges with recruitment may have arisen because staff, often surgeons, were reportedly worried about 'looking stupid' or being criticised in front of their team.

'The one we did here with ENT, all four of my colleagues came up to me separately, to ask 'is it going to be okay?' As a surgeon you really don't like looking stupid and you really don't like being out of control.'

(DHB3, surgeon)

Instructor participants described tackling this challenge by reassuring colleagues that the training was not an assessment, and that the focus was on teamwork. Other instructor participants felt that approaching a surgeon to volunteer for a course required courage. Positive word of mouth about the course also helped motivate attendance by staff less willing to participate. Participants often described handpicking the early course attendees, to encourage a ripple-on effect of enthusiasm, while in one DHB they had success tackling the tricky departments first.

'Our strategy is to engage with the engaged people. I don't think me talking to a surgeon will particularly motivate him to do it, but talking to another surgeon will. So, training those who are already keen, and getting them to spread the word.' (DHB1, anaesthetist)

'So in May (it) was general surgeons and then we focused on ENT and orthopaedics. Those departments have sometimes been the difficult departments to get engagement with. So, we actually targeted them specifically because we felt that, well if we can get them on board the rest will just naturally come on board and the enthusiasm and the shift has been fantastic.' (DHB4, manager)

\section{Learning}

Participants attributed the success of NetworkZ to the learning environment created by the in situ scenarios, the high quality of the simulations and the approaches to learning. Instructors identified debriefing as critical to the success of training, but many felt anxious about their own expertise as debriefers. While some staff accepted that their skills would evolve over time, others were anxious about debriefing senior colleagues and staff from other disciplines. Nurse participants in particular, discussed their concerns about debriefing.

'I don't' find that instructing my nurses, but I do feel a little bit, you know, like there's a difference instructing surgeons and anaesthetists, I find that quite different, I don't have the same confidence.' (DHB2, nurse)

Some participants expressed concern about debriefing their more forceful colleagues who were openly critical of NetworkZ. However, they noted that it many of these sceptics were won over by training.

'We've had some real negative vibes of people starting the course who've done a complete turnaround and said actually they were really glad they'd done it, said it was really good. And we've had some very positive feedback from people who were quite sceptical.' (DHB2, surgeon).

'We have a couple of sceptics in the ENT Department and they all turned up and we were actually pleasantly surprised because in the end they were all converted, they were like actually this is really good.' (DHB3, manager)

Participants who had witnessed effective NetworkZ debriefing, and seen its positive impact on groups after training, indicated an appreciation of the techniques and how they flattened hierarchies, facilitated learning and simply made teams more talkative with one another.

'When debriefing it's like they're all on the same level, there's no real hierarchy sort of thing. I think it's good for building team relationships as well.' (DHB2, nurse)

'They have noticed that we aren't that great at communicating at times and it's helped them realise where we could improve our communication.' (DHB5, anaesthetist)

Some participants said they were wary of the challenges of role conflict when training their own staff, and some felt that external facilitators would be more respected by course attendees. One participant suggested debriefers could travel between DHBs.

\section{Motivation}

Participants were enthusiastic about NetworkZ and excited to be part of its implementation. The participants felt motivated to implement NetworkZ as it aligned with their intrinsic interest in promoting safety and effective communication. They valued the rich evidence-base underpinning the NetworkZ programme, and the expertise of the programme developers. They used words such as 'exciting' and 'empowered' to describe their involvement in NetworkZ.

'I wanted to do something positive and exciting ... and I quite like that it's research based as well and there's good evidence for it.' (DHB3, surgeon) 
'When I do a NetworkZ course I know that they will go away and feel empowered enough to change something.' (DHB5, anaesthetist)

Many managers also welcomed NetworkZ because of perceived benefits to the quality and safety of work in the OT. 'We're investing in safe practice within the theatre, we're investing in making sure that our systems are as effective and as thorough as they should be.' (DHB5, management). Some managers were excited about taking on the work of change management, and others hoped that similar programmes could be offered throughout the hospital. These motivational strengths were particularly important when staff encountered implementation barriers including resistance from detractors, and competing demands on their time.

Local ownership of the programme was a key aspect of the programme for some participants.

'This is about theatre. It's not about anybody else, it's about how we can engage and improve our systems of work. It's not someone from the outside saying, 'no this is how you're going to change it'.' (DHB4, nurse)

There was an evident will to persist and ensure the success of the programme. However, we did observe that some staff also showed signs of weariness due to significant commitments of personal time, or slow progress in implementation. This suggested that ongoing support is needed to maintain staff enthusiasm and commitment.

\section{DISCUSSION}

NetworkZ is an ambitious quality improvement project. It is large scale and resource intensive. It challenges existing practices in uni-disciplinary continuing professional development. Furthermore, it is disruptive, pitting the needs for daily service delivery against the needs for team training. A programme of this scale requires a sound approach to implementation. Our study identified elements of our early implementation that were working, and those that required modification or strengthening for ongoing implementation of the programme in subsequent cohorts.

\section{What we learnt about structure}

Without the appropriate DHB structure for course implementation, the motivation of implementation staff was tested, training was delayed and OT staff who disliked the idea of training easily avoided participation. Dixon-Woods et $a l^{15}$ warn us that ongoing structural support is key to sustainability of improvement programmes. So too do McCulloch et $a l,{ }^{17}$ who noted that the transfer of learning from team training into OT practice may be impossible without continuous direct promotion and institutional support. Paull et $a l^{18}$ similarly found that facility leadership was the strongest predictor of the implementation of perioperative team training. Our findings reflect this. Management support for implementation was essential to free up staff and theatre time for the training. Rhee et al discovered that closing operating rooms to do training has tremendous symbolic power. ${ }^{19}$ Closing theatres for NetworkZ training symbolised that teamwork and staff were valued in their organisation.

A lesson for the future is the need for ongoing engagement with senior management. While all senior management groups signed up to the programme, their ongoing support was variable. The benefits of the training, apparent to those delivering it, were perhaps less obvious to senior and middle management. Strengthening the links between the teams delivering the training, and management is necessary. Formally requiring multidisciplinary team training as a key performance indicator for healthcare organisations may ultimately be necessary for long-term sustainability of such programmes.

The success of implementation often depends on the personalities and credibility of implementation leaders and managers, existing interpersonal histories and people's previous experiences with similar programmes. We identified concerns or fears that deterred some from active participation in NetworkZ. This type of locally run simulation training is challenging. It requires clear communication about the purpose of the training, the nature of the course and the shared responsibility for learning. As such, we are improving the pre-course information, and explicitly addressing this concept of mutual respect and shared responsibility for learning in the introduction to the course.

\section{What we learnt about infrastructure}

There is some debate about the value of creating physical high fidelity simulations in team training. ${ }^{20}$ While fidelity is resource intensive, our study participants considered fidelity an essential element of NetworkZ, and a key factor in attracting and enthusing surgical participants. This affirmed our investment in bespoke interactive surgical models and providing the DHBs with their own simulator.

\section{What we learnt about culture}

The key finding from this research was that existing culture was both a challenge and the driving force behind the effectiveness and success of NetworkZ implementation. Participants were positive about the implementation of NetworkZ because they saw it as driving a necessary cultural transformation in their OTs towards improved communication, staff relationships, attitudes, workflow and teamwork. Participants saw NetworkZ as both a programme (tool for change) and a process (of cultural change), and this mirrors findings concerning the Surgical Safety Checklist as both tool and process. ${ }^{21}$ Linking multidisciplinary team training to existing safety interventions and organisational safety frameworks therefore seems a useful approach. Our collaboration and co-branding of materials with the HQSC, a national body tasked with improving quality and safety in NZ hospitals is likely to have enhanced the acceptance of the programme as a credible quality improvement initiative. 
A somewhat unexpected finding was that staff perceived NetworkZ as creating an opportunity for them to improve on workplace relationships. With the current international focus on toxic workplaces, hierarchical and repressive team leadership, bullying and harassment, ${ }^{22}$ this finding may prove to be an important lever for the implementation of NetworkZ or similar interventions.

\section{What we learnt about politics}

Establishing multidisciplinary training is political. For it to occur, all groups need to participate on an equal footing. This challenges traditional hierarchies, and traditional approaches to professional development, and generates challenges for recruitment of participants to attend the training. We sought the support of key professional bodies, which may have helped to overcome some of the local political barriers. When implementing NetworkZ in future cohorts, we intend to focus more on providing the type of evidence valued by the different groups, for example, improved efficiencies, improved patient throughput, improved patient outcomes may be of key importance to some groups, while improved workplace interactions and morale may be more important to others.

\section{What we learnt about learning}

Our aim was for local instructors to run NetworkZ independently within 12 months. We were perhaps too optimistic about the speed at which local instructors would acquire the competencies and confidence to run the programme. We have modified our implementation processes to provide centralised support for local DHBs instructors for a longer period of time. For some DHBs, perhaps due to their small size, we consider ongoing external support may be required, either from a central hub, or from other DHBs with more resources and expertise. We are also considering modifications to our instructor training approaches, to facilitate the development of debriefing skills.

We identified anxiety among instructors regarding these skills. Debriefing has been described at length in the literature, with emphasis often on its phases or approaches to questioning. ${ }^{23} 24$ There is little guidance on the complexity of debriefing colleagues, with existing social, hierarchical and professional relationships. If instructors and participants' interactions are coloured by previous history, trust may potentially be more difficult to establish. Strategies to approach this require future development, but at this point, we have incorporated a focussed discussion on this as part of our instructor training. Another option is to swap instructors between DHBs to mitigate this phenomenon.

\section{What we learnt about motivation}

We identified an intrinsic motivation of participants to improve the culture in the workplace, and their skills in teamwork and communication, and a belief that NetworkZ could achieve this. Further, NetworkZ simulations are fun and novel, in contract to regulation-focused quality improvement programmes and thus staff enthusiasm was relatively easy to generate, once initial scepticism was overcome.

We also identified that resistance, lack of support, or over-commitment could dampen enthusiasm. We have identified the need for ongoing instructor recruitment and training, as well as an overarching national strategy to build further support for the programme.

\section{LIMITATIONS}

Interview participants were volunteers who had agreed both to the interview, and to taking part in the implementation of the NetworkZ programme in their hospital, and were likely to be invested in its success. This study has only reported on the perspectives of local hospital staff charged with the implementation of the programme, and has not included the perspectives of those charged with the overall implementation at the national level. The extent to which implementation lessons from a programme in New Zealand apply to other populations remains to be tested.

\section{Recommendations for large-scale implementation of team training}

New Zealand's small population and single government create an environment in which things can happen quite quickly on a national scale. The extent to which this programme can be scaled up to larger populations and more complex health systems is for others to test. We do think, however, there are some lessons for others.

- We believe a key component of success for NetworkZ is the fact that it is recognised as a national programme and supported by national institutions.

- Local ownership and delivery of the team training programme, with ongoing support from a central organisation, looks to be an effective implementation strategy.

- Support is needed at multiple levels, and needs to be continually renewed and reinforced.

- The culture change necessary to introduce multidisciplinary training should not be underestimated.

- Programme quality is key. We consider the quality of the simulations, designed to engage all participants, is crucial. Stakeholders across the board need to be convinced by the training.

- Evidence for the training needs to be provided, initially from other similar initiatives but, as evidence is collected, from the programme itself.

- An exhaustive and ongoing communication strategy at all levels is essential to maintain senior management support for implementation.

\section{CONCLUSION}

We identified challenges and successes for implementation of the NetworkZ programme that will inform strategies for implementation in subsequent cohorts of 
DHBs across NZ. The potential gains from a national, multidisciplinary OT team training intervention such as NetworkZ are substantial, in terms of improved culture in the workplace, improved teamwork and communication and anticipated effects on patient safety. However, embedding such programmes effectively in multiple sites across a nation requires significant and sustained support at all levels to ensure its ongoing success.

\section{Consent}

All participants in this study voluntarily provided written consent prior to their participation. All people identifiable in figure 2 have provided written consent to the publication of the image.

\section{Author affiliations}

${ }^{1}$ Centre for Medical and Health Sciences Education, The University of Auckland, Auckland, New Zealand

${ }^{2}$ Department of Surgery, University of Auckland, Auckland, New Zealand

${ }^{3}$ Surgery, Auckland City Hospital, Auckland, New Zealand

${ }^{4}$ Department of Anaesthesia, Capital and Coast District Health Board, Wellington, New Zealand

${ }^{5}$ Department of Anaesthesia and Perioperative Medicine, Auckland City Hospital, Auckland, New Zealand

${ }^{6}$ Department of Anaesthesiology, The University of Auckland, Auckland, New Zealand

Contributors All authors (TJ, PB, JL, IC, AG, AFM, CS, JT, JW) made substantial contributions to the study design. PB and CS collected and coded the data. Data coding was checked by JW, KH, TJ. PB and TJ analysed the data. TJ, JW, PB and $\mathrm{JL}$ drafted the manuscript. All authors contributed to subsequent iterations and approved the final manuscript.

Funding This research was funded by Accident Compensation Corporation New Zealand, Lotteries Health Research Fund New Zealand, and the Australia and New Zealand College of Anaesthetists. ANZCTR trial number: ACTRN12617000017325.

Competing interests Alan F Merry is Chair of the Health Quality and Safety Commission, New Zealand.

Patient consent for publication Not required.

Ethics approval This research was approved on 3 October 2016 by the Northern B Health and Disability Ethics Committee (16/NTB/143). Separate approval was also provided locally by each District Health Board.

Provenance and peer review Not commissioned; externally peer reviewed.

Data availability statement № data are available.

Open access This is an open access article distributed in accordance with the Creative Commons Attribution Non Commercial (CC BY-NC 4.0) license, which permits others to distribute, remix, adapt, build upon this work non-commercially, and license their derivative works on different terms, provided the original work is properly cited, appropriate credit is given, any changes made indicated, and the use is non-commercial. See: http://creativecommons.org/licenses/by-nc/4.0/.

ORCID iDs

Tanisha Jowsey http://orcid.org/0000-0003-1499-9225

Jennifer Weller http://orcid.org/0000-0002-8752-9286

\section{REFERENCES}

1 Jha AK, Larizgoitia I, Audera-Lopez C, et al. The global burden of unsafe medical care: Analytic modelling of observational studies. BMJ Qual Saf 2013;22:809-15.
2 de Vries EN, Ramrattan MA, Smorenburg SM, et al. The incidence and nature of in-hospital adverse events: a systematic review. Quality and Safety in Health Care 2008;17:216-23.

3 Lingard L, Espin S, Whyte S, et al. Communication failures in the operating room: an observational classification of recurrent types and effects. Qual Saf Health Care 2004;13:330-4.

4 Kohn LT, Corrigan JM, Donaldson MS. To err is human: building a safer health system. National Academies Press 2000.

5 Leape LL, Berwick DM. Five years after to err is human: what have we learned? JAMA 2005;293:2384-90.

6 Hughes AM, Gregory ME, Joseph DL, et al. Saving lives: a meta-analysis of team training in healthcare. J Appl Psychol 2016;101:1266-304.

7 Jones KJ, Skinner AM, High R, et al. A theory-driven, longitudinal evaluation of the impact of team training on safety culture in 24 hospitals. BMJ Qual Saf 2013;22:394-404.

8 Salas E, Zajac S, Marlow SL. Transforming health care one team at a time: ten observations and the TRAIL ahead. Group Organ Manag 2018;43:357-81.

9 Weller J, Cumin D, Torrie J, et al. Multidisciplinary operating room simulation-based team training to reduce treatment errors: a feasibility study in New Zealand hospitals. $N Z$ Med $J$ 2015;128:40-51.

10 Ministry of Health. District health boards. 19 March 2019.. Available: https://www.health.govt.nz/new-zealand-health-system/key-healthsector-organisations-and-people/district-health-boards

11 Ministry of Health. Services delivered: acute and elective patient discharge volumes. Available: https://www.health.govt.nz/ publication/services-delivered-acute-and-elective-patient-dischargevolumes [Accessed 07 May 2019].

12 Health Quality \& Safety Commission New Zealand. Safe surgery NZ. Available: https://www.hqsc.govt.nz/our-programmes/safe-surgery$\mathrm{nz} /$ [Accessed 03 Oct 2018].

13 Braithwaite J, Runciman WB, Merry AF. Towards safer, better healthcare: harnessing the natural properties of complex sociotechnical systems. Qual Saf Health Care 2009;18:37-41.

14 Bate P, Mendel P, Robert G. Organizing for Quality: The Improvement Journeys of Leading Hospitals in Europe and the United States. Oxford and New York: Radcliffe Publishing, 2008.

15 Dixon-Woods M, McNicol S, Martin G. Ten challenges in improving quality in healthcare: lessons from the health Foundation's programme evaluations and relevant literature. BMJ Qual Saf 2012;21:876-84.

16 Gale NK, Heath G, Cameron E, et al. Using the framework method for the analysis of qualitative data in multi-disciplinary health research. BMC Med Res Methodol 2013;13:117-24.

17 McCulloch P, Mishra A, Handa A, et al. The effects of aviation-style non-technical skills training on technical performance and outcome in the operating theatre. Qual Saf Health Care 2009;18:109-15.

18 Paull DE, Mazzia LM, Izu BS, et al. Predictors of successful implementation of preoperative briefings and postoperative debriefings after medical team training. Am J Surg 2009;198:675-8.

19 Rhee AJ, Valentin-Salgado Y, Eshak D, et al. Team training in the perioperative arena: a methodology for implementation and auditing behavior. Am J Med Qual 2017;32:369-75.

20 Hamstra SJ, Brydges R, Hatala R, et al. Reconsidering fidelity in simulation-based training. Acad Med 2014;89:387-92.

21 Mitchell B, Cristancho S, Nyhof BB, et al. Mobilising or standing still?A narrative review of Surgical Safety Checklist knowledge as developed in 25 highly cited papers from 2009 to 2016 . BMJ Qual Saf 2017;26:837-44.

22 Lever I, Dyball D, Greenberg N, et al. Health consequences of bullying in the healthcare workplace: a systematic review. $J$ Adv Nurs. In Press 2019. doi:10.1111/jan.13986. [Epub ahead of print: 28 Feb 2019].

23 Rudolph JW, Simon R, Raemer DB, et al. Debriefing as formative assessment: closing performance gaps in medical education. Acad Emerg Med 2008;15:1010-6.

24 Cheng A, Eppich W, Grant V, et al. Debriefing for technologyenhanced simulation: a systematic review and meta-analysis. Med Educ 2014;48:657-66. 